\title{
Panic Attacks Induced by Doxapram
}

\author{
Yue-Joe Lee, George C. Curtis, John G. Weg, James L. Abelson, \\ Jack G. Modell, and Kelly M. Campbell
}

\section{Introduction}

Doxapram is a respiratory stimulant thought to act by stimulating medullary neurones and carotid oxygen receptors (Kato and Buckley 1964). It changes neither oxygen uptake nor $\mathrm{CO}_{2}$ production, but produces substantial hyperventilation (Calverley et al 1983).

Hyperventilation occurs during naturally occurring or pharmacologically induced panic attacks, and perhaps also in panic patients between attacks (Gorman et al 1984, 1986). In panic disorder, central respiratory centers (Woods et al 1988) or a "suffocation alarm" center (Klein in press) may be hyperexcitable. We report here a pilot study designed to test the possibility that in panic discrder these centers may be hypersensitive to doxapram.

\section{Subjects and Methods}

Subjects of the study were (1) five patients (4 women, 1 man, mean age 32 years, range-19-47) with DSM-III-R panic disorder with or without agoraphobia; (2) five normal controls (all women, mean age 34 years, range-28-39); and (3) three male psychiatrists (age 63, 36, and 34) who were investigators in the study. Status of patients and controls was determined by structured interview, medical and psychiatric history, and physical examination. Subjects were excluded for heavy use of caffeine, tobacco, alcohol, or other substance abuse or any significant medical condition. All were medication free for at least 1 month. Subjects were informed that they would receive a placebo and a doxapram injection, without knowing when they were to occur. They were told that doxapram would cause hyperventilation, tachycardia, and a hot sensation in the head and trunk, lasting about 5 min. Written consent was obtained. Noninvestigator subjects were paid $\$ 50$.

On the scheduled day, patients skipped lunch and at about 2

From the Department of Psychiatry, National Taiwan University, Taipeh. Taivan (Y-JL); Department of Psychiatry (GCC, JLA), Department of Internal Medicine (JGW, KMC), University of Michigan, Ann Arbor, MI; and Department of Psychiatry, University of Alabama, Tuscaloosa, AI. (JGM).

Address reprint requests to George C. Curtis, MD, Department of Psychiatry, University of Michigan, Med Inn 444, 1500 East Medical Center Drive. Ann Arbor, Michigan $18109-0840$.

Received May 6, 1992; revised October 12, 1992.

Presented in part at American Psychiatric Association, New Orleans, Louisiana May 11-16, 1991, and American College of Neuropsychopharmacology, San Juan, Puerto Rico, December 9-13, 1991.
PM were placed in a comfortable reclining chair. A butterfly needle was placed in a dorsal hand vein and connected to a slow, normal saline drip. Electrocardiographic leads were connected for continuous recording. A nose clip was applied, and the subject breathed through a soft plastic mouthpiece connected to a CAD*NET SYSTEM 2001 (Medical Graphics Corp., St. Paul, MN) permitting breath-by-breath analysis of ventilation and gas exchange. Three consecutive monitoring periods of approximately 5-15 min were carried out. Nothing was administered during the first period, which served for measuring and extinguishing responses to the novel situation. During the second period, a saline injection was administered through the intravenous tubing, and during the third, doxapram $0.5 \mathrm{mg} / \mathrm{kg}$ body weight was administered over $15 \mathrm{sec}$ by the same route. Injections were given single blind. The psychiatrist-subjects did not undergo adaptation or placebo periods.

Prior to the adaptation period, subjects were interviewed about their subjective state at that moment, including moods, feelings, thoughts, bodily sensations, and ratings of intensity of global distress and individual panic symptoms. After baseline stabilization of physiological measures following each injection, subjects were again interviewed and gave similar ratings about the preceding period. A panic attack was scored if: (a) the subject called the experience a panic attack; and (b) rated four or more DSM-III-R panic attack symptoms as at least "moderate" on a 4-point scale of "not at all," "mild," "moderate," and "severe" and (c) rated the experience as 5 or more on a 7-point scale where 1 was "no similarity" and 7 was "identical" to a naturally occurring panic attack. Criterion (c) was omitted for nonpatient subjects. Subjects also gave 0 to 100 anijogue ratings for subjective units of distress (SUD) at each interview.

Physiologic measures were averaged over consecutive 1-min epochs. Patients and controls were compared at each time point by unpaired $t$-tests. One-tailed tests of significance at $p<0.05$ were employed.

\section{Results}

Following doxapram, every subject described a hot sensation, usually beginning in the perineum, then radiating over the trunk and head. Self-ratings of distress and total severity ratings of panic attack symptoms were higher in patients than in controls at all time points, significantly so during the adaptation and doxapram periods. No subject met panic attack criteria during the adaptation or placebo periods. Following doxapram, four of five patients, one 
of five controls, and none of the three psy'chiatrist-subjects met the specified criteria for a panic attack ( $p<0.03$ excluding the psychiatrists, $p<0.01$ including them). Heart rate was similar in patients and controls during adaptation and placebo periods and increased markedly following doxapraii, with nonsignificant trends toward greater increase in patients. Respiratory rate, tidal volume, and minute ventilation increased markedly in both groups following doxapram. The following were significantly greater in patients postdoxapram: respiratory rate at $4 \mathrm{~min}$; tidal volume at 5 and 8 min; minute ventilation at 4 and $5 \mathrm{~min}$. As shown in Figure 1, end tidal $\mathrm{pCO}_{2}$ was lower in patients than in controls at all time points, the differences being significant during adaptation and at $\min 3$ through 9 postdoxapram.

\section{Discussion}

Aside from the known effects of doxapram, the most robust findings were the greater anxiogenic (including panicogenic) ef- fect in patients and the larger ventilatory response, manifested most robustly by respiratory alkalosis. The greater ventilatory response could be either a cause or a result of the greater anxiogenic effects. As with other panicogenic probes, these could be caused by either group differences in biological sensitivity to the drug or to cognitively mediated differences in fear of the somatic sensations caused by the drug. The biological possibilities are numerous, but include group differences in metabolism of doxapram and differential sensitivities of respiratory control or "suffocation alarm" systems as outlined earlier. In a small pilot study such as this, a number of issues remain unaddressed. The possibility that the findings were caused by chance variation and small sample size must be considered. Whether doxapram might trigger panic attacks in other disorders has not been explored, nor is it known whether repeat challenge in patients with panic disorder would prouluce simitán resuits. Adaditional research is necessary in order to resolve these issues. The results to date suggest that doxapram may be an additional useful probe for investigating the pathophysiology of panic disorder.

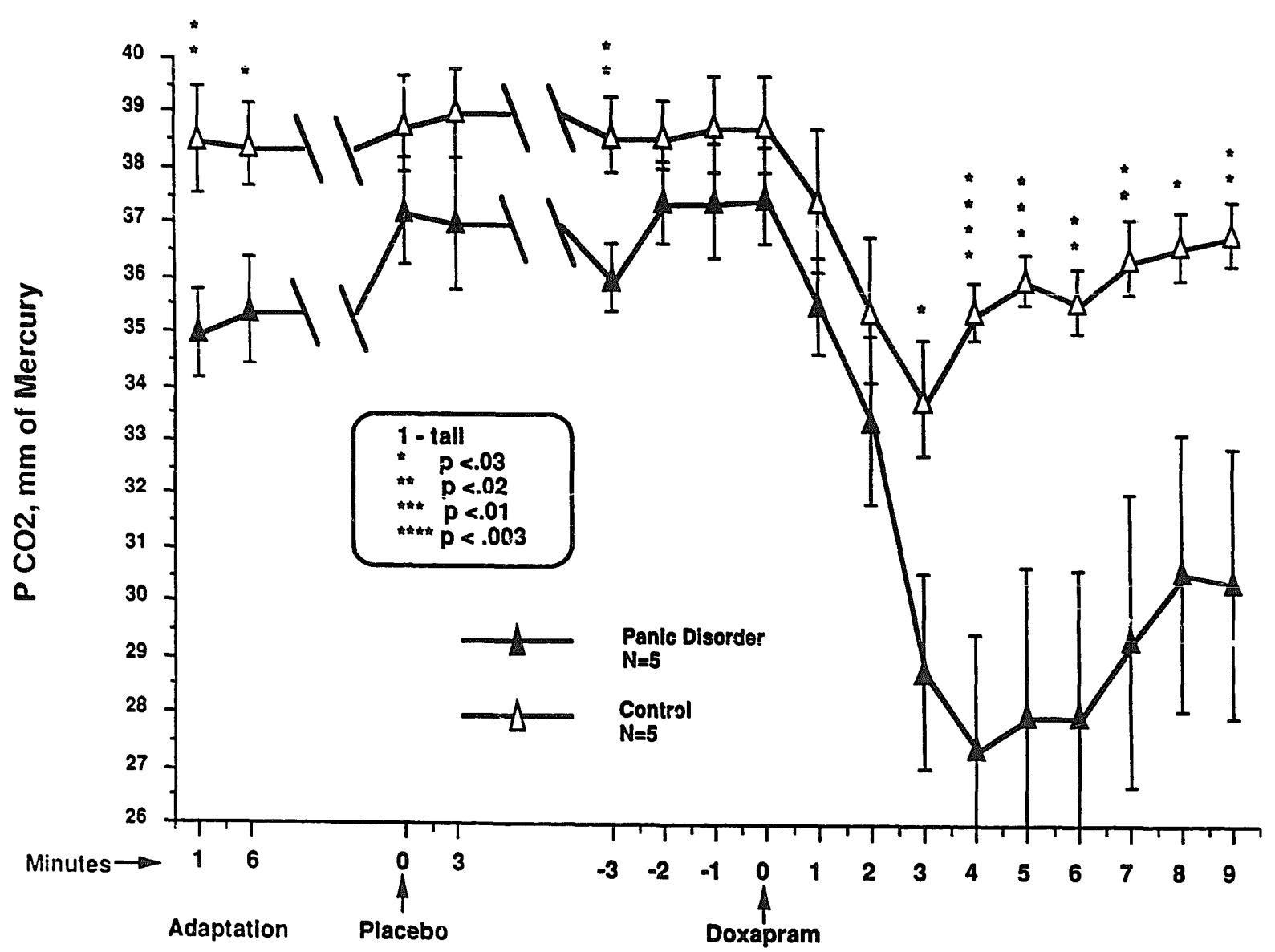

Figure 1. Response of panic patients and control subjects to laboratory adaptation, placebo administration, and doxapram. End tidal $\mathrm{pCO}_{2}, \pm \mathrm{SEM}$. 


\section{References}

Calverley PMA, Robson RH, Wraith PK, Prescott LF, Flenley DC (1983): The ventilatory effects of doxapram in normal man. Clin Sci 65:65-69.

Gorman JM, Askanazi J, Liebowitz MR, et al (1984): Response to hyperventilation in a group of patients with panic disorder. Am J Psychiatry 141:857-861.

Gorman JM, Cohen BS, Liebowitz MR, et al (1986): Blood gas changes and hypophosphatemia in lactate-induced panic. Arch Ger: Psychiatry 43:1067-1071.
Kato H, Buckley JP (1964): Possible sites of action of the respiratory stimulant effect of doxapram hydrochloride. J Pharmacol Exp Ther 144:262-264.

Klein DF (in press): False suffocation alarms and spontaneous panics: Subsuming the $\mathrm{CO} 2$ hypersensitivity theory. Arch Gen Psychiatry.

Woods SW, Charney DS, Goodman WK (1988): Carbon dioxideinduced anxiety. Arch Gen Psychiatry 45:43-52. 